\title{
Of Dogs and Fleas: The Dynamics of $N$ Uncoupled Two-State Systems
}

\author{
Ch. Hauert, ${ }^{1}$ J. Nagler, ${ }^{2}$ and H. G. Schuster ${ }^{3}$
}

Received May 26, 2003; accepted February 4, 2004

\begin{abstract}
The historical Ehrenfest model dating back to 1907 describes the process of equilibration together with fluctuations around the thermal equilibrium. This approach represents a special case in the dynamics of $N$ uncoupled two-state systems. In this article we present a generalization of the original model by introducing an additional parameter $p$ which denotes the probability of a single state change. Analytical solutions for the probability distribution of the system's state as well as the fluctuation distribution are derived. Interestingly, close inspection of the fluctuation distribution reveals an intrinsic time scale. Sampling the system's state at much slower rates yields the familiar macroscopic exponential distribution for equilibrium processes. For faster measurements a power law extends roughly over $\log _{10} N$ orders of magnitude followed by an exponential tail. At some point, further increases of the sampling rate merely result in a shift of the fluctuation distribution towards higher values leaving plateau at small fluctuation sizes behind. Since the generic solution is rather unwieldy, we derive and discuss simple and intuitive analytical solutions in the limit of small $p$ and large $N$. Furthermore, we relax the quantization of time by considering a complementary approach in continuous time. Finally we demonstrate that the fluctuation distributions resulting from the two different approaches bear identical characteristic features.
\end{abstract}

KEY WORDS: Equilibrium physics; levelling processes; thermal processes; random walks.

\footnotetext{
${ }^{1}$ Departments of Zoology \& Mathematics, University of British Columbia, 6270 University Boulevard, Vancouver B.C. V6T 1Z4, Canada.

${ }^{2}$ Institut für Theoretische Physik, Fachbereich 1, Universität Bremen, Otto-Hahn-Allee, D-28334 Bremen, Germany; e-mail: nagler@itp.uni-bremen.de

${ }^{3}$ Institut für Theoretische Physik und Astrophysik, Christian-Albrechts-Universität, Olshausenstraße 40, D-24118 Kiel, Germany.
} 


\section{INTRODUCTION}

The flea model by Ehrenfest ${ }^{(1,2)}$ essentially describes the jumps of $N$ fleas between two dogs. In each time step a single randomly selected flea jumps on the other dog. The more fleas there are on a dog, the more will jump off it. In the long time limit and in absence of external tuning, the mean number of fleas on each dog approaches the equilibrium value $N / 2$. Thus, the Ehrenfest model describes the details of the equilibration process together with fluctuations around the thermal equilibrium.

The relevance of such slow leveling processes for the power law behavior of self-organizing systems has been emphasized and quantified in earlier works. ${ }^{(3,4)}$ In particular, the model proposed in ${ }^{(4)}$ essentially reduces to the Ehrenfest model. To put this in a more general context, we consider the generic dynamics of $N$ uncoupled two-state systems. Despite their abundance in nature and widespread physical relevance (we just mention stochastic resonance with applications to bistable systems as lasers and optical traps, see review ${ }^{(5)}$ and references therein) we were unable to find this straightforward generalization in the literature.

In the original model, Ehrenfest assumed that in each time step exactly one flea jumps. This rather restrictive assumption had the fortunate tradeoff to yield simple solutions. Here we demonstrate that an analytical solution is still attainable in the more general case where all fleas jump with a certain probability $p$. At the same time this represents an important step towards relaxing Ehrenfest's assumption of discrete time events. From this formulation we can not only recover the original model for particular jump probabilities $p$ but also derive a continuous time formulation.

\section{THE MODEL}

Suppose that $\operatorname{dog} A$ is harassed by $N_{A}$ fleas and $\operatorname{dog} B$ by $N_{B}=N-N_{A}$. In every time step each flea jumps with a probability $p$ to the other dog and remains on its victim with $1-p$. In the long run, the average flea load is equal for each $\operatorname{dog} N_{A}=N_{B}=N / 2$. But their numbers fluctuate over time. A single fluctuation is defined as a process that starts once $N_{A}$ becomes larger than $N / 2$ and stops when it gets back to it for the first time. The same holds for $N_{B}$. Thus, the end of one fluctuation specifies the start of the subsequent one. For simplicity, we assume $N$ even throughout the text. For $N$ odd, the definition of the fluctuations has to be changed accordingly. The duration or length $\lambda$ of a fluctuation is determined by the number of time steps elapsed until the fluctuation ends. 
Let us first determine the time evolution of the probability to find $N_{A}$ fleas on $\operatorname{dog} A$ which is determined by a master equation:

$$
P^{\tau+1}\left(N_{A}\right)=\sum_{n=0}^{N} a_{N_{A}, n} P^{\tau}(n)
$$

with

$$
\begin{aligned}
a_{N_{A}, n}= & (1-p)^{N}\left(\frac{p}{1-p}\right)^{n-N_{A}} \\
& \times \sum_{i=0}^{N_{A}}\left(\begin{array}{c}
N_{A} \\
i
\end{array}\right)\left(\begin{array}{c}
N-N_{A} \\
n-N_{A}+i
\end{array}\right)\left(\frac{p}{1-p}\right)^{2 i},
\end{aligned}
$$

where $a_{N_{A}, n}$ specifies the transition probability from $n$ fleas on $\operatorname{dog} A$ at time $\tau$ to $N_{A}$ in the next time step. Note that throughout the article binomial coefficients with negative entries are assumed to be zero.

Surprisingly, this rather awkward equation is easily solved when returning to the picture of $N$ uncoupled two-state systems. Each such system consists of a single flea sitting on either dog. The probability $p$ to switch dogs is simply the transition probability between the two states.

For a moment, lets consider only a single flea: $Q^{\tau}(s)$ denotes the probability to find the flea on $\operatorname{dog} s$ at time $\tau$. For convenience we choose $s \in\{0,1\}$. The dynamics of this one-flea-system is then given by another master equation:

$$
Q^{\tau+1}(s)=(1-p) Q^{\tau}(s)+p Q^{\tau}(1-s)
$$

It follows that

$$
Q^{\tau+1}(s)-Q^{\tau+1}(1-s)=(1-2 p)^{\tau+1}\left[Q^{0}(s)-Q^{0}(1-s)\right],
$$

and finally

$$
Q^{\tau}(s)=\frac{1}{2}\left[1-(1-2 p)^{\tau}\right]+(1-2 p)^{\tau} Q^{0}(s),
$$

where we used the fact that the flea must reside on either dog, i.e. $Q^{\tau}(s)+Q^{\tau}(1-s)=1$. Thus, the probability to find a particular system in state 1 (on $\operatorname{dog} A$, say) at time $\tau$ is then given by: 


$$
\begin{aligned}
& q_{0}(\tau)=\frac{1}{2}\left(1-(1-2 p)^{\tau}\right), \\
& q_{1}(\tau)=\frac{1}{2}\left(1+(1-2 p)^{\tau}\right),
\end{aligned}
$$

where $q_{i}(\tau)$ applies depending on the initial state $i=0,1$. Obviously $\sum_{i} q_{i}(\tau)=1$ holds since each flea must have originated in either one of the dogs.

Equipped with Eq. (2), we return to $N$ fleas. Assuming that initially $N_{A}^{0}$ fleas were sitting on $\operatorname{dog} A$, the solution of Eq. (1) can be readily derived. We simply have to add up all possibilities to end up with $N_{A}$ fleas at time $\tau$ when starting with $N_{A}^{0}$. Using the time dependent probabilities of Eq. (2) we obtain:

$$
\begin{aligned}
P_{N_{A}^{0}}^{\tau}\left(N_{A}\right)= & \sum_{i=0}^{N_{A}}\left(\begin{array}{l}
N_{A}^{0} \\
i
\end{array}\right) q_{1}(\tau)^{i}\left(1-q_{1}(\tau)\right)^{N_{A}^{0}-i} \\
& \times\left(\begin{array}{l}
N-N_{A}^{0} \\
N_{A}-i
\end{array}\right) q_{0}(\tau)^{N_{A}-i}\left(1-q_{0}(\tau)\right)^{N-N_{A}^{0}-N_{A}+i} \\
= & q_{1}(\tau)^{N}\left(\frac{q_{0}(\tau)}{q_{1}(\tau)}\right)^{N_{A}+N_{A}^{0}} \\
& \times \sum_{i=0}^{N_{A}}\left(\begin{array}{l}
N_{A}^{0} \\
i
\end{array}\right)\left(\begin{array}{l}
N-N_{A}^{0} \\
N_{A}-i
\end{array}\right)\left(\frac{q_{1}(\tau)}{q_{0}(\tau)}\right)^{2 i} .
\end{aligned}
$$

Note that $i$ out of the $N_{A}$ fleas originate from $\operatorname{dog} A$ and the remaining $N_{A}-i$ fleas from $\operatorname{dog} B$. In the long time limit $\tau \rightarrow \infty$ we obtain the expected binomial distribution which is independent of the initial condition $N_{A}^{0}$ :

$$
P^{\infty}\left(N_{A}\right)=2^{-N}\left(\begin{array}{l}
N \\
N_{A}
\end{array}\right)
$$

From Eqs. (3), (4) and the well-known mirror-method ${ }^{(6)}$ (see Fig. 1) the fluctuation distribution $D(\lambda)$ is obtained. First we need to derive the probabilities that in the next time step a new fluctuation starts or the current ends. Using the mirror-method we then determine all allowed trajectories connecting the starting and ending points of a fluctuation of given length $\lambda$. Finally, the weighted sum over all trajectories yields $D(\lambda)$.

In order to keep things simple, we assume that the system has reached its equilibrium distribution $P^{\infty}\left(N_{A}\right)$ at time $\tau=0$. Consequently, the 


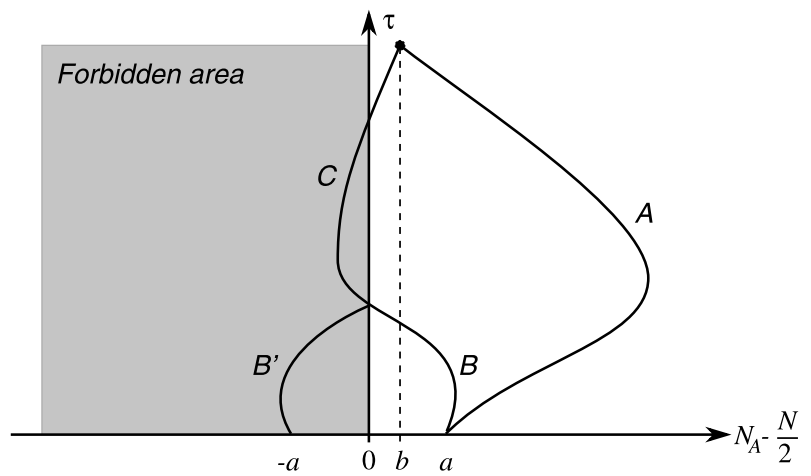

Fig. 1. The mirror method: $A$ indicates a valid path, connecting $N_{A}=N / 2+a$ and $N / 2+b$. $B C$ represents a forbidden path because it crosses $N / 2$. However note that each such path $B$ has a counterpart of equal probability $B^{\prime}$ starting in $N_{A}=N / 2-a$.

probability for the start of a new fluctuation at $\tau=1$ with initial size $a$ is given by

$$
f(a)=\sum_{a^{\prime}=0}^{N / 2} P_{a^{\prime}}^{1}(N / 2+a) P^{\infty}\left(a^{\prime}\right)
$$

Similarly, the probability that the current fluctuation with $N_{A}=N / 2+b$ ends in the next time step is given by

$$
g(b)=\sum_{a^{\prime}=0}^{N / 2} P_{N / 2+b}^{1}\left(a^{\prime}\right)
$$

To connect the start and end points of one fluctuation, we have to determine all valid trajectories and their probabilities. This means we consider all paths starting in $N_{A}=N / 2+a$ at time $\tau=1$ with $a \in\{1, \ldots, N / 2\}$ and ending in $N_{A}=N / 2+b$ at time $\tau=\lambda-1$ which do not cross $N / 2$. This probability is calculated by the mirror method (see Fig. 1). It states that each invalid path has a counterpart of equal probability starting in $N_{A}=$ $N / 2-a$. Therefore we consider all paths and subtract the invalid ones, i.e. those starting in $N_{A}=N / 2-a$. The corresponding probabilities are simply given by Eq. (3). Finally, we sum over all starting and ending points $a$ and $b$ weighted by the corresponding frequencies $f(a)$ and $g(b)$ :

$$
D(\lambda)=2 \sum_{a=1}^{N / 2} \sum_{b=0}^{N / 2} f(a)\left(P_{N / 2+a}^{\lambda-2}(b)-P_{N / 2-a}^{\lambda-2}(b)\right) g(b),
$$




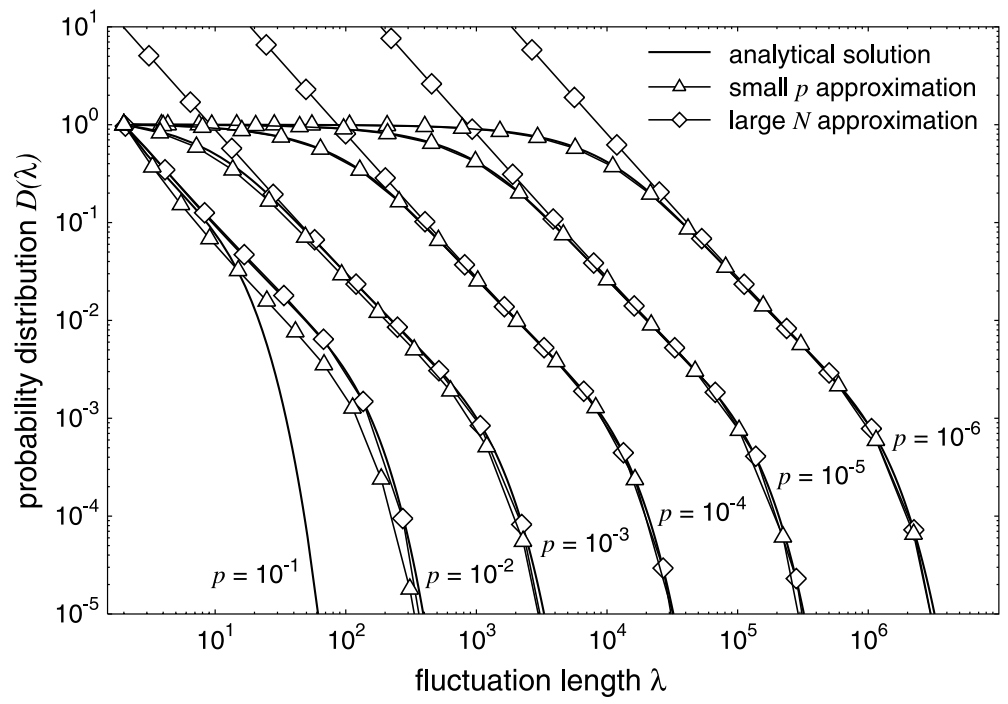

Fig. 2. Fluctuation distribution derived by numerical evaluations of Eq. (7) for $N=100$ fleas and different jump rates $p$ (solid lines). Generally, the distribution is divided into three regimes: a flat plateau for small $\lambda$, an intermediate range governed by a power law and an exponential tail for large $\lambda$. Decreasing $p$ essentially shifts the distribution towards larger fluctuation sizes while increasing the plateau. $(\triangle)$ indicate an approximation derived in the limit of small $p$ (see Eq. (17)). The quality of the approximation obviously increases with decreasing $p$. $(\diamond)$ denote another approximation derived in the continuum limit $N \rightarrow \infty$ (see Eq. (20)). However, the plateau occurring for finite $N$ is missing. All distributions have an arbitrary normalization such that $D(2)=1$.

for $\lambda \geq 2$. The factor two in Eq. (7) results from the symmetry of the system. Numerical evaluations of Eq. (7) are shown for different jump rates $p$ in Fig. 2 and different numbers of fleas $N$ in Fig. 3. Both figures clearly show three distinct regimes of $D(\lambda)$ :

(i) For small $\lambda \lesssim 1 /(p N)$ a plateau is observed and $D(\lambda)$ remains approximately constant. We will argue that this region contains little information and results from the time resolution of the measurement.

(ii) For intermediate lengths $1 /(p N) \lesssim \lambda \lesssim 1 / p$ a power law distribution is observed indicating scaling invariance of the fluctuation lengths. The width of the power law regime scales with the system size $N$ and roughly extends over $\log _{10} N$ orders of magnitude.

(iii) For large $\lambda \gtrsim 1 / p$ the distribution has an exponential tail due to finite size effects. In the limit $N \rightarrow \infty$ this tail vanishes. 


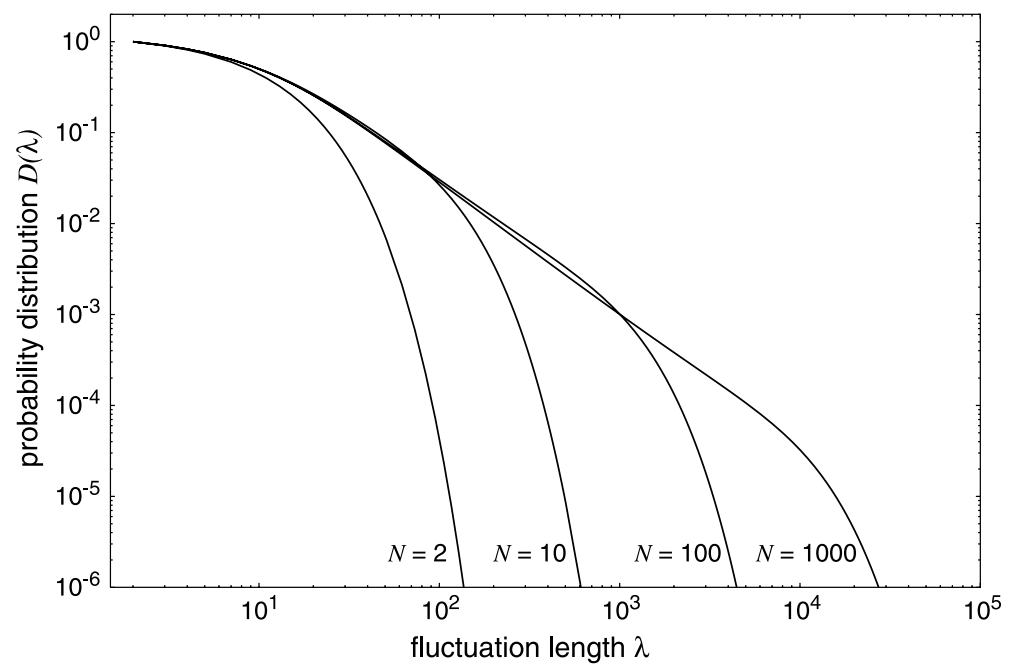

Fig. 3. Fluctuation distribution derived through numerical evaluations of Eq. (7) for different numbers of fleas $N$ and jump rates $p=0.1 / N$. The power law regime of the distribution clearly scales with $N$ and roughly extends over $\log _{10} N$ orders of magnitude. All distributions have an arbitrary normalization such that $D(2)=1$.

Figure 2 demonstrates that decreasing the jump rate $p$ with $N=$ const. essentially results in a shift of $D(\lambda)$ to larger fluctuation sizes while increasing the plateau. Note that changes of $p$ correspond to a linear transformation of the time resolution of the measurement $\Delta \tau \rightarrow \Delta \tau / p N$. Thus, the model has an intrinsic time scale defined by $\Delta \tau \sim 1 / p N$. Measuring the state at this rate results in complete information on the dynamical properties and no plateau is observed (c.f. Fig. 2 for $N=100$ and $p=$ $1 / N=0.01$ ). Increasing the measurement rate to $\Delta \tau \lesssim 1 / p N$ only results in an increased plateau for small $\lambda$. On the other hand, for slower measurements with $\Delta \tau \gtrsim 1 / p N$ information is lost and eventually we are left with the exponential tail. Consequentially, power law behavior of fluctuations may be observed in any leveling process for appropriate time resolutions.

Qualitatively similar observations were made in an experimental setup measuring the avalanche distributions in rice piles ${ }^{(7,8)}$. The measured distributions crucially depend on the geometrical properties of the rice grains, i.e., their friction. For large frictions, related to small jump rates $p$, a power law distribution including a plateau is observed, while small frictions, corresponding to larger $p$, resulted in a (stretched) exponential distribution. 


\section{CONTINUOUS TIME}

The somewhat artificial discretization of time in the Ehrenfest model can be avoided $a b$ initio by assuming a probability density function

$$
f(t)=k e^{-k t}
$$

which determines the jump probability of a single flea, i.e. in the time interval $d t$ the flea jumps with probability $f(t) d t$, and satisfies the normalization condition $\int_{0}^{\infty} f(t) \mathrm{d} t=1$. The constant $k$ relates to the half-life of a flea on one dog and is thus related to the jump probability $p$ in discrete time. As a first step, we need to derive the probability $P_{n}(t)$ that a single flea jumps exactly $n$ times up to time $t$. Therefore we split the interval $[0, t]$ into $M$ equal subintervals. For sufficiently large $M$, i.e. in the limit $M \rightarrow \infty$, the flea either stays in place or makes a single jump in every subinterval. The jump probability during each subinterval is given by:

$$
\int_{0}^{t / M} f(t) \mathrm{d} t=1-e^{-k t / M}
$$

Putting this together to get exactly $n$ jumps in the $M$ intervals yields

$$
\begin{aligned}
P_{n}(t) & =\lim _{M \rightarrow \infty}\left(\begin{array}{c}
M \\
n
\end{array}\right)\left(1-e^{-k t / M}\right)^{n}\left(e^{-k t / M}\right)^{M-n} \\
& =e^{-k t} \lim _{M \rightarrow \infty}\left(\begin{array}{c}
M \\
n
\end{array}\right)\left(e^{k t / M}-1\right)^{n} \\
& =\frac{e^{-k t}}{n !} \lim _{M \rightarrow \infty} \frac{M !}{(M-n) !}\left[\sum_{j=1}^{\infty}(k t)^{j} M^{-j}\right]^{n},
\end{aligned}
$$

where $\mathcal{P}=M ! /(M-n)$ ! represents a polynomial in $M$ of order $n$. The limit $M \rightarrow \infty$ eliminates all terms involving $\mathcal{O}\left(M^{n-1}\right)$ of $\mathcal{P}$ :

$$
P_{n}(t)=\frac{e^{-k t}}{n !}(k t)^{n}
$$

Now we are ready to derive a counterpart to Eqs. (2) in continuous time by noting that finding a flea on one dog (state 1) at time $t$ requires an even number of jumps if the flea sat initially on that dog (state 1) and an odd number of jumps if the flea was on the other $\operatorname{dog}$ (state 0 ) at $t=0$ : 


$$
\begin{aligned}
& q_{0}^{\mathrm{c}}(t)=\sum_{n=0}^{\infty} P_{2 n+1}(t)=\frac{1}{2}\left(1-e^{-2 k t}\right), \\
& q_{1}^{\mathrm{c}}(t)=\sum_{n=0}^{\infty} P_{2 n}(t)=\frac{1}{2}\left(1+e^{-2 k t}\right) .
\end{aligned}
$$

The upper index in $q_{i}^{\mathrm{c}}(t)$ marks that these formulas refer to the continuous time formulation.

In order to simplify comparisons between the discrete and continuous approach, we set $k=-\frac{1}{2} \log (1-2 p)$ with $0 \leq p<\frac{1}{2}$. This converts Eqs. (11) into

$$
\begin{aligned}
& q_{0}^{\mathrm{c}}(t)=\frac{1}{2}\left(1-(1-2 p)^{t}\right), \\
& q_{1}^{\mathrm{c}}(t)=\frac{1}{2}\left(1+(1-2 p)^{t}\right)
\end{aligned}
$$

which are formally equivalent to Eqs. (2). From this it is straight forward to derive the analogue to Eq. (3) in continuous time:

$$
\begin{aligned}
P_{N_{A}^{0}}^{t}\left(N_{A}\right)= & q_{1}^{\mathrm{c}}(t)^{N}\left(\frac{q_{0}^{\mathrm{c}}(t)}{q_{1}^{\mathrm{c}}(t)}\right)^{N_{A}+N_{A}^{0}} \\
& \times \sum_{i=0}^{N_{A}}\left(\begin{array}{l}
N_{A}^{0} \\
i
\end{array}\right)\left(\begin{array}{l}
N-N_{A}^{0} \\
N_{A}-i
\end{array}\right)\left(\frac{q_{1}^{\mathrm{c}}(t)}{q_{0}^{\mathrm{c}}(t)}\right)^{2 i} .
\end{aligned}
$$

Equipped with Eq. (13) we are now able to derive the fluctuation distribution $D_{\mathrm{c}}(l)$ in continuous time following a similar line of argument as in discrete time. Assume that the system is in equilibrium with $N_{A}=N / 2$. Further, let us for a moment focus our attention on fluctuations that are accompanied with states on the right half plane, i.e. $N / 2 \leq N_{A} \leq N$ only. As soon as one additional flea reaches $\operatorname{dog} A$ another avalanche starts. But note that in each infinitesimal time interval $d t$ at most a single flea jumps. Thus, the initial size of an avalanche in continuous time is always 1, i.e. all avalanches start with $N / 2+1$. For the same reason, an avalanche that is about to end will also have size 1, i.e. $N_{A}=N / 2+1$. Assuming that the avalanche started at time $t=0$ we now need to determine the probability that the system is again in state $N / 2+1$ at time $t=l$ without reaching $N / 2$ before, i.e. that an avalanche of length $l$ has occurred. This is again accomplished by the mirror method:

$$
D_{\mathrm{c}}(l)=C_{N, p}\left(P_{N / 2+1}^{l}(N / 2+1)-P_{N / 2-1}^{l}(N / 2+1)\right),
$$


where $C_{N, p}$ accounts for the probability that the avalanche actually ends after reaching $N / 2+1$ at time $l$, i.e. the transition probability $N / 2+$ $1 \rightarrow N / 2$, as well as a factor 2 arising from the symmetry of the system. It is important to note that $C_{N, p}$ is independent of the duration of the avalanche and is hence determined by the normalization condition $\int_{0}^{\infty} D_{\mathrm{c}}(l) d l=1$.

Due to the formal equivalence of Eq. (3) and Eq. (13) the corresponding fluctuation distributions for the time continuous case Fig. 2 and Fig. 3. are essentially identical to those depicted in Fig. 2 and Fig. 3 for discrete time (not shown).

\section{APPROXIMATIONS}

In discrete time the exact analytical solution (7) is unfortunately quite unwieldy. Thus, aiming at simple and intuitive representations, we derive two approximations of Eq. (7) by first considering the limit of small $p$ and then turning to the continuum limit $N \rightarrow \infty$.

\section{Small Jump Rates}

The original master Eq. (1) is greatly simplified in the limit of small jump rates $p \leqslant 1 / N$. In this case, we may assume that at most one flea jumps per time step and Eq. (1) becomes:

$$
\begin{aligned}
P^{\tau+1}\left(N_{A}\right)= & p\left(N_{A}+1\right) P^{\tau}\left(N_{A}+1\right) \\
& +p\left(N-N_{A}+1\right) P^{\tau}\left(N_{A}-1\right) \\
& +(1-p N) P^{\tau}\left(N_{A}\right) .
\end{aligned}
$$

Upon setting $p=1 / N$ Eq. (15) reduces to the original Ehrenfest model with well-known analytical solutions for $P^{\tau}\left(N_{A}\right)$ and $D(\lambda)^{(9)}$. However, following $\operatorname{Kac}^{(9)}$ (see appendix) the more general case Eq. (15) for arbitrary $p$ can be solved for the initial condition $P^{0}\left(N_{A}\right)=\delta\left(N_{A}-N_{A}^{0}\right)$ :

$$
P^{\tau}\left(N_{A}\right)=(-1)^{N+N_{A}^{0} 2^{-N}} \sum_{j=0}^{N}(1-2 p j)^{\tau} C_{N_{A}}^{j} C_{j}^{N-N_{A}^{0}}
$$

where the $C_{k}^{j}$ s are defined through the identity

$$
(z-1)^{j}(z+1)^{N-j}=\sum_{k=0}^{N} C_{k}^{j} z^{k} .
$$


The fluctuation distribution $D(\lambda)$ is derived as before by applying the mirror method and setting $a=b=1$ :

$$
\begin{aligned}
D^{\prime}(\lambda)= & \frac{p^{2} N(N+2)}{2} \\
& \times\left(P_{N / 2-1}^{\lambda-2}(N / 2-1)-P_{N / 2+1}^{\lambda-2}(N / 2-1)\right) .
\end{aligned}
$$

Inserting Eq. (16) yields

$$
\begin{aligned}
D^{\prime}(\lambda)= & p^{2} N(N+2)(-1)^{N / 2+1} 2^{-N-1} \\
& \times \sum_{j=0}^{N}(1-2 p j)^{\lambda} C_{N / 2-1}^{j}\left[C_{j}^{N / 2+1}-C_{j}^{N / 2-1}\right]
\end{aligned}
$$

for $N$ even, $\lambda \geq 2$ and $0<p \leq 1 / N$. For small jump rates $p$ Eq. (17) is a very good approximation to the general solution Eq. (7) as shown in Fig. 2.

\section{Continuum Limit}

Another and more elegant approximation is obtained in the continuum limit $N \rightarrow \infty$ and for small $p$. Introducing the scaling variables $x, t, q$ and the probability density $f(x, t)$

$$
\begin{aligned}
x & =\left(N_{A}-N / 2\right) / \sqrt{N}, \\
t & =\tau / N, \\
q & =p N, \\
f(x, t) & =N P^{\tau}\left(N_{A}\right)
\end{aligned}
$$

Eq. (15) leads to a Fokker-Planck equation:

$$
\partial_{t} f(x, t)=q\left(\frac{1}{2} \partial_{x}^{2}+2 \partial_{x} x\right) f(x, t) .
$$

Eq. (18) describes a random walker in a parabolic potential $V(x)=q x^{2}$. The solution of Eq. (18) is found in the literature, given that the system was in state $x_{0}$ at time $t=0$, i.e. $f(x, 0)=\delta\left(x-x_{0}\right)$ :

$$
f(x, t)=\frac{1}{\sqrt{\pi}} \sigma(t)^{-1} \exp \left(-\frac{\left(x-x_{0} e^{-2 q t}\right)^{2}}{\sigma(t)^{2}}\right),
$$


with

$$
\sigma(t)^{2}=\frac{1}{2}\left(1-e^{-4 q t}\right)
$$

The fluctuation distribution $D(l)$ with $l=\lambda / N$ then specifies the probability that the random walker in $V(x)$ leaves the origin $x=0$ at time $t$ and returns for the first time at $t+l$. Note that $D(l)$ corresponds to the time evolution of the flux leaving the system at an absorbing boundary in $x=0$. A solution of Eq. (18) with an absorbing boundary in $x=0$ is generated by differentiating Eq. (19) with respect to $x_{0}{ }^{(4)}$. Using the continuity equation we then calculate the flux $j(x, t)$ in $x=0$ :

$$
j(x, t)=-\int \partial_{t} g(x, t) d x .
$$

This finally leads to $D(l)$ for fluctuations starting in $x=0$ at time $t=0$ and ending in $x=0$ at $t=l$ :

$$
D(l)=\sqrt{\frac{8}{\pi}}\left(1-e^{-4 q l}\right)^{-\frac{3}{2}} e^{-2 q l},
$$

denoting a power law distribution with an exponential tail due to finite size effects. $D(l)$ becomes exact and a pure power law for $N \rightarrow \infty$. At the same time, Eq. (20) is incapable of reproducing the plateau for small l. However, note that from Eq. (17) follows that for $l \ll 1 /(q N)$ (or $\lambda \ll$ $1 /(p N))$ the fluctuation distribution remains approximately constant, i.e.

$$
D(l) \propto \begin{cases}\text { const. } & \text { if } l \lesssim 1 /(q N) \\ \left(1-e^{-4 q l}\right)^{-\frac{3}{2}} e^{-2 q l} & \text { if } l \gtrsim 1 /(q N)\end{cases}
$$

which resembles a low pass filter (see Fig. 2).

\section{DISCUSSION}

The Ehrenfest model describes the equilibration process and fluctuations in thermal equilibrium of $N$ uncoupled two-state systems under the rather artificial and restrictive assumption that in each time step exactly one state change occurs. In the present article we relax this assumption by introducing the parameter $p$ which denotes the probability of a state change for each two-state system. In this generalized framework, Ehrenfest's model represents a special case for $p=1 / N$. Despite the additional 
complications, analytical solutions for the probability distribution and the corresponding fluctuation distribution are still attainable.

The fluctuation distribution generally shows three distinct regimes: (a) a plateau for small fluctuations, (b) a power law regime for intermediate and (c) an exponential tail for large fluctuations. However, the power law regime is observed only for sufficiently small jump rates $p \lesssim 1 / N$. Its width scales with $N$ and extends roughly over $\log _{10} N$ orders of magnitude. Larger $p$ leave only the exponential tail while smaller $p$ increase the size of the plateau.

An equivalent condition for observing power law behavior in any leveling process refers to the required resolution of measurements: $\Delta t \sim 1 / p N$. Slower sampling rates would reveal only the exponential tail - in accordance with macroscopic equilibration processes - while higher rates do not lead to more accurate information but merely produce larger plateaus.

The Ehrenfest model and its more general variant are essentially equivalent to a random walk in a parabolic potential. In the former case the walker makes a step to the left or right with certainty while in the latter he remains in place with probability $1-2 p$. The potential not only drives the walker back to the origin, i.e. the system to its equilibrium state, but it also explains in a natural way the exponential tail of the fluctuation distribution arising from finite size effects.

Apart from simple and intuitive approximations in the limit of small $p$ and large $N$ we provide a complementary approach in continuous time producing identical characteristic features of the fluctuation distribution.

Two state systems, where these effect should be observable, are abundant in nature ${ }^{(5)}$. We just mention Ising magnets in the paramagnetic state where deviations of the magnetization from zero should display fluctuations whose durations are distributed with a power law - at least for sufficiently fast measurements.

\section{APPENDIX: SOLUTION OF THE SIMPLIFIED SYSTEM}

In order to derive a solution for Eq. (15), i.e. in the limit of small $p$, we follow the approach of $\mathrm{Kac}^{(9)}$ to solve the original Ehrenfest model.

The initial condition for the probability distribution is given by $P^{0}\left(N_{A}\right)=\delta\left(N_{A}-N_{A}^{0}\right)$, where $N_{A}^{0}$ specifies the number of fleas on $\operatorname{dog} A$ at time $\tau=0$ and $\delta$ denotes the Kronecker delta.

To find $P^{\tau}\left(N_{A}\right)$, we use the method of Markov chains. Let $(p)_{\tau}$ be the vector 


$$
(p)_{\tau}=\left(\begin{array}{l}
P^{\tau}(0) \\
P^{\tau}(1) \\
\vdots \\
P^{\tau}(N)
\end{array}\right)
$$

at time $\tau$ and $A$ the $(N+1) \times(N+1)$ matrix

$$
A=\left(\begin{array}{llll}
1-p N & p & 0 & 0 \\
p N & 1-p N & 2 p & 0 \\
0 & p(N-1) & 1-p N & 0 \\
0 & 0 & p(N-2) & 0 \\
\vdots & & & \ddots \\
0 & 0 & 0 & \ldots 1-p N
\end{array}\right)
$$

Thus, the master equation Eq. (15) can be written in matrix form as

$$
(p)_{\tau+1}=A(p)_{\tau}
$$

and it follows that

$$
(p)_{\tau}=A^{\tau}(p)_{0}
$$

where the vector $(p)_{0}$ represents the initial condition with all zeros except the $N_{A}^{0}$ th component which is one. $P^{\tau}\left(N_{A}\right)$ is then the element $\left(N_{A}, N_{A}^{0}\right)$ of $A^{\tau}$.

The matrix $A$ can be diagonalized as follows:

$$
A=P\left(\begin{array}{cccc}
\lambda_{0} & 0 & \ldots & 0 \\
0 & \lambda_{1} & & 0 \\
\vdots & & \ddots & 0 \\
0 & 0 & \ldots & \lambda_{N}
\end{array}\right) Q
$$

where $P Q=1$ and $\lambda_{j}$ being the $N+1$ eigenvalues of the matrix $A$. Using Eq. (21) we calculate the right and left eigenvectors. Let $C^{0}, C^{1}, \ldots, C^{N}$ be the right and $\alpha_{0}, \alpha_{1}, \ldots, \alpha_{N}$ the left (normalized) eigenvectors to the eigenvalues $\lambda_{0}, \lambda_{1}, \ldots, \lambda_{N}$, respectively. For $j=0,1, \ldots, N$ we then have

$$
\begin{aligned}
A C^{j} & =\lambda_{j} C^{j}, \\
A^{T} \alpha_{j} & =\lambda_{j} \alpha_{j},
\end{aligned}
$$


where $A^{T}$ denotes the transpose of $A$. The orthonormalization condition reads

$$
C^{j} \alpha_{k}=\delta(j, k)
$$

where $\delta(j, k)$ denotes Kronecker's delta. The eigenvalues $\lambda_{j}$ and the corresponding right eigenvectors are determined by a system of $N+1$ linear equations:

$$
\lambda x_{k}=p(N-k+1) x_{k-1}+(1-p N) x_{k}+(k+1) p x_{k+1},
$$

with $k=0,1, \ldots, N$. To solve Eq. (23), we use the method of generating functions. Multiplying the members of Eq. (23) by $1, z, z^{2}, \ldots, z^{N}$, and summing up, we obtain

$$
\begin{gathered}
p \sum_{k=1}^{N}(N-k+1) x_{k-1} z^{k}+\sum_{k=0}^{N}(1-p N) x_{k} z^{k} \\
+p \sum_{k=0}^{N}(k+1) x_{k+1} z^{k}=\lambda \sum_{k=0}^{N} x_{k} z^{k} .
\end{gathered}
$$

Introducing the abbreviation

$$
f(z)=\sum_{k=0}^{N} x_{k} z^{k}
$$

we get

$$
f^{\prime}(z)=\frac{\lambda-1+p N(1-z)}{p\left(1-z^{2}\right)} f(z)
$$

which is a linear differential equation for the generating function $f(z)$. It's solution reads

$$
f(z)=x_{0}(-1)^{\frac{1-\lambda}{2 p}}(z-1)^{\frac{1-\lambda}{2 p}}(z+1)^{\frac{\lambda-1+2 p N}{2 p}}
$$

and satisfies the condition $f(0)=x_{0}$.

The $N+1$ eigenvalues of $A$ are now easily obtained:

$$
\lambda_{j}=1-2 p j, \quad \text { for } \quad j=0,1, \ldots, N .
$$


Since $f(z)$ is a polynomial of degree $N$, it follows that the components of the right eigenvectors $C_{0}^{j}, C_{1}^{j}, \ldots, C_{N}^{j}$ to the eigenvalues $\lambda_{j}=1-2 p j$ are defined by the identity

$$
(z-1)^{j}(z+1)^{N-j}=\sum_{k=0}^{N} C_{k}^{j} z^{k}
$$

In order to calculate the left eigenvectors $\alpha_{j}^{k}$ we use of Eq. (22) such that

$$
\begin{aligned}
z^{j} & =\sum_{r=0}^{N} \delta(j, r) z^{r}=\sum_{r=0}^{N}\left(\sum_{k=0}^{N} C_{r}^{k} \alpha_{k}^{j}\right) z^{r} \\
& =\sum_{k=0}^{N} \alpha_{k}^{j}\left(\sum_{r=0}^{N} C_{r}^{k} z^{r}\right) .
\end{aligned}
$$

Using Eq. (24) yields

$$
z^{j}=(z+1)^{N} \sum_{k=0}^{N} \alpha_{k}^{j}\left(\frac{z-1}{z+1}\right)^{k} .
$$

Let

$$
\xi=\frac{z-1}{z+1}
$$

then Eq. (25) reads in terms of $\xi$ :

$$
\sum_{k=0}^{N} \alpha_{k}^{j} \xi^{k}=2^{-N}(1+\xi)^{j}(1-\xi)^{N-j}
$$

Comparing Eqs. (26) and (24) we get

$$
\alpha_{j}^{k}=(-1)^{N-k} 2^{-N} C_{j}^{N-k} .
$$

Finally, the solution of the master equation Eq. (15) is obtained from Eq. (21):

$$
P^{\tau}\left(N_{A}\right)=(-1)^{N+N_{A}^{0}} 2^{-N} \sum_{j=0}^{N}(1-2 j p)^{\tau} C_{N_{A}}^{j} C_{j}^{N-N_{A}^{0}} .
$$




\section{ACKNOWLEDGMENTS}

We thank an anonymous referee for pointing out the continuous time approach based on Eq. (8). Ch.H. acknowledges support of the Swiss National Science Foundation 8220-64682.

\section{REFERENCES}

1. P. and T. Ehrenfest, Phys. Z. 8:311 (1907).

2. H.G. Schuster Complex Adaptive Systems: An Introduction (Scator Verlag, Saarbrücken, 2002).

3. H. Flyvbjerg, Phys. Rev. Lett. 76:940 (1996).

4. J. Nagler, C. Hauert and H. G. Schuster, Phys. Rev. E 60:2706 (1999).

5. L. Gammaitoni, P. Hänggi, P. Jung, and F. Marchesoni, Rev. Mod. Phys. 70:223 (1998).

6. M. E. Fisher, J. Stat. Phys. 34:667 (1984).

7. V. Frette, K. Christensen, A. Malthe-Sørensen, J. Feder, T. Jøssang, and P. Meakin, Nature 379:49 (1996).

8. K. Christensen, Á. Corral, V. Frette, J. Feder, and T. Jøssang, Phys. Rev. Lett. 77:107 (1996).

9. M. Kac, Random Walk and Theory of Brownian motion, American Math. Monthly, Vol. 54 no. (1947), e.g. in Selected Papers on Noise and Stochastic Processes, Dover Publications, New York (1954). 